\title{
A system-level mechanistic investigation of traditional Chinese medicine, Yinlai Decoction, for related diseases
}

\author{
Tie-Gang Liu, Zi-An Zheng, Yu-Xiang Wan, Chen Bai, Jing-Nan Xu, He Yu and \\ Xiao-Hong Gu* \\ School of Basic Medical Sciences, Beijing University of Chinese Medicine, Beijing 100029, China
}

*For correspondence: Email: greatscenery@126.com; Tel/Fax: 008610153562

Sent for review: 27 March 2017

Revised accepted: 20 July 2017

\begin{abstract}
Purpose: To systemically explore the pharmacological mechanisms of traditional Chinese medicine, Yinlai Decoction (YD), used in the clinical management of pediatric diseases such as pneumonia and recurrent respiratory tract infections.

Methods: An ingredient-target-disease database of YD was constructed using Traditional Chinese Medicine Systems Pharmacology Database and Analysis Platform (TCMSP). First, the molecular targets related to lung and stomach diseases were searched and screened to avoid duplication. Second, the associations between these molecular targets were evaluated via Search Tool for the Retrieval of Interacting Genes/Proteins (STRING) and Gene Ontology (GO) and Pathway enrichment analysis in STRING.

Results: A total of 627 chemical ingredients and 654 protein targets in YD were obtained. After further screening, 38 molecular targets linked to respiratory diseases, inflammatory responses and various infections were identified. Finally, 576 GO terms and 75 KEGG pathway terms were obtained by analyzing gene functional annotation clusters and abundance value of these targets. Most of these terms were closely related to the inflammatory response.

Conclusion: Based on these in silico findings, the use of $Y D$ for treating respiratory diseases, inflammation and various infections, most probably via the suppression of inflammation, has been established. The approach adopted in this study can serve as a model methodology to develop an innovative TCM candidate drug at a network pharmacology level.
\end{abstract}

Keywords: Yinlai Decoction, Network (System) pharmacology, Inflammation, Interacting genes/proteins, Gene ocntology, Pathway enrichment analysis

Tropical Journal of Pharmaceutical Research is indexed by Science Citation Index (SciSearch), Scopus, International Pharmaceutical Abstract, Chemical Abstracts, Embase, Index Copernicus, EBSCO, African Index Medicus, JournalSeek, Journal Citation Reports/Science Edition, Directory of Open Access Journals (DOAJ), African Journal Online, Bioline International, Open-J-Gate and Pharmacy Abstracts

\section{INTRODUCTION}

Yinlai Decoction (YD) is an empirical formula used to treat lung and stomach diseases, especially pneumonia and recurrent respiratory tract infections in children. This traditional formula is composed of Lonicerae japonicae Flos, Raphani semen, Forsythiae fructus, Scutellariae radix, Houttuyniae herba, Peucedani radix and Trichosanthes kirilowii Maxim [1].
Biological mechanisms of YD have been related to the regulation of expression of inflammatory factors, such as tumor necrosis factor- $\alpha$ (TNF- $\alpha$ ) and interleukin-6 (IL-6) [2-4]. Moreover, the existing experimental data is either fragmented or confined to a single index or one pathway, thus it cannot reflect the overall multi-target regulation of this traditional Chinese medicine's characteristics. 
On account of this deficiency in knowledge, we have introduced system theory as an attempt to explore the possible mechanism of YD. Network pharmacology is an emerging discipline, which is useful to study the progression of disease, the interaction of drug with the body and the discovery of new drugs from the perspective of biological networks [5]. The basic idea of network pharmacology is to intervene the pathological network of disease, rather than just individual genes associated with disease, to achieve a comprehensive prevention and treatment effects.

By analyzing the existing databases, we sorted out all chemical ingredients present in YD. Targets of all these chemical molecules were identified, and then the related pathways or diseases for each target were annotated. Through correlation and pathway analysis of targets related to the lung and stomach diseases treatable with YD, we predicted network-based mechanism of YD for related diseases.

\section{METHODS}

\section{Database construction}

Traditional Chinese Medicine Systems Pharmacology Database and Analysis Platform (TCMSP, http://lsp.nwsuaf.edu.cn/tcmsp.php) is a unique platform of system pharmacology for Chinese herbal medicines that that renders a relationship between drugs, molecular targets and diseases states. This database is not only accompanying with various pharmacological networks, but also exhibits pharmacokinetic features of natural drugs such as solubility, permeability and bioavailability. This advancement has initiated a new thematic search to find candidate drugs in different types of traditional Chinese herbs [6]. All chemicals of each herb and all targets of each chemical were retrieved through TCMSP and the available literature. Specific retrieval steps were: (i) retrieval of YD ingredients; (ii) target search of each chemical, then (iii) building of chemicaltargets database of YD.

\section{Target identification}

A target-disease database was developed after retrieval of the related diseases of each target through TCMSP. Afterwards, the corresponding molecular targets of these chemical ingredients were identified for further analysis.

\section{Network construction and analysis}

For systematic investigation of mechanism of YD, the interaction between these molecular targets were analyzed through Search Tool for the Retrieval of Interacting Genes / Proteins (STRING, http://string-db.org/). STRING database is an excellent source of hundreds of known interactions between proteins as well as is capable of predicting interactions. This database retrieves the knowledge of both physical (direct) and functional (indirect) interactions from four different sources of information such as genomic literature, high-throughput screening, coexpression and the published information. STRING combines the interaction data from these sources for hundreds of organisms, and transfers information between these organisms where applicable. This database presently covers $9,643,763$ proteins from 2,031 organisms [7].

The relationship between herbs and the screened targets was analyzed by constructing target-herb network to investigate the mechanism of YD and its significance of pharmacodynamic composition. The network was generated by Cytoscape 3.4.0 [8].

\section{Gene ontology and pathway enrichment analysis}

In order to identify and analyze the specific biological properties of the potential targets, Gene Ontology (GO) biological processes were introduced to dissect target genes in a hierarchically structured way based on the characteristic biological terms. Pathway enrichment analysis was introduced to probe the mechanisms of YD for related diseases. Both GO terms and KEGG pathway terms were obtained from STRING. Then, more information of these pathways were acquired from Kyoto Encyclopedia of Genes and Genomes (KEGG, http://www.kegg.jp/) database.

\section{RESULTS}

\section{Herb-compound-target database}

A total of 236 molecular compounds in Lonicerae japonicae Flos and 388 potential targets, 52 molecular compounds in Raphani semen and 103 targets, 80 molecular compounds in Forsythiae fructus and 323 targets, 58 molecular compounds in Scutellariae radix and 197 targets, 208 molecular compounds in Houttuyniae herba and 50 targets, 101 molecular compounds in Peucedani radix and 369 targets, and 150 molecular compounds and 362 targets in Trichosanthes kirilowii Maxim were obtained. Chemical compounds and their targets were merged to remove overlapping. As a result, 627 
molecular compounds and 654 potential targets in YD were found.

\section{Targets and the related diseases}

According to early experimental results and clinical applications, YD could be used to treat inflammation, respiratory diseases and bacterial and viral infections. Out of 654 targets of YD, 38 targets related to these diseases screened out and mapped to the database UniProt (http://www.uniprot.org/) for normalization.

Out of these 38,33 protein targets were found to exist in Homo sapiens (Table 1). Betalactamases ( $\beta$-lactamases) are enzymes (EC 3.5.2.6) produced by bacteria that provide resistance to $\beta$-lactam antibiotics such as the penicillins, cephamycins, and carbapenems.

Table 1: Molecular targets of the retrieved targets and their respective disease information in Homo sapiens

\begin{tabular}{|c|c|c|c|}
\hline $\begin{array}{l}\text { UniProt } \\
\text { ID }\end{array}$ & Target & $\begin{array}{c}\text { Gene } \\
\text { names }\end{array}$ & Diseases \\
\hline P35354 & Prostaglandin $\mathrm{G} / \mathrm{H}$ synthase 2 & PTGS2 & Inflammation, Inflammatory diseases \\
\hline P23219 & Prostaglandin $\mathrm{G} / \mathrm{H}$ synthase 1 & PTGS1 & Chronic inflammatory diseases \\
\hline P05231 & Interleukin-6 & IL6 & Inflammatory Disorders, Unspecified \\
\hline P29474 & Nitric-oxide synthase, endothelial & NOS3 & Inflammation; Sepsis \\
\hline P07550 & Beta-2 adrenergic receptor & ADRB2 & $\begin{array}{l}\text { Inflammation; Chronic obstructive pulmonary } \\
\text { disease, unspecified }\end{array}$ \\
\hline P01375 & Tumor necrosis factor & TNF & $\begin{array}{l}\text { Chronic inflammatory diseases; Periodic fever } \\
\text { syndrome }\end{array}$ \\
\hline Q02880 & DNA topoisomerase II & TOP2B & Bacterial Infections; Herpes virus infection \\
\hline P08172 & $\begin{array}{l}\text { Muscarinic acetylcholine receptor } \\
\text { M2 }\end{array}$ & CHRM2 & $\begin{array}{l}\text { Chronic obstructive pulmonary disease, } \\
\text { unspecified }\end{array}$ \\
\hline$\underline{\mathrm{P} 37231}$ & $\begin{array}{l}\text { Peroxisome proliferator activated } \\
\text { receptor gamma }\end{array}$ & PPARG & Chronic inflammatory diseases, Inflammation \\
\hline P09960 & Leukotriene A-4 hydrolase & LTA4H & Inflammation \\
\hline P09917 & Arachidonate 5-lipoxygenase & ALOX5 & $\begin{array}{l}\text { Inflammatory bowel disease; Inflammatory lung } \\
\text { disease }\end{array}$ \\
\hline Q03181 & $\begin{array}{l}\text { Peroxisome proliferator activated } \\
\text { receptor delta }\end{array}$ & PPARD & Inflammation \\
\hline P13500 & C-C motif chemokine 2 & CCL2 & $\begin{array}{l}\text { Chronic obstructive pulmonary disease, } \\
\text { unspecified; Inflammatory diseases associated } \\
\text { with hypoxia }\end{array}$ \\
\hline P01584 & Interleukin- 1 beta & IL1B & Acne; Pediatric \\
\hline P15692 & Vascular endothelial growth factor $\mathrm{A}$ & VEGFA & Viral infection; Herpes virus infection \\
\hline P35372 & Mu-type opioid receptor & OPRM1 & Cough \\
\hline P11926 & Ornithine decarboxylase & ODC1 & Inflammation \\
\hline Q13085 & Acetyl-CoA carboxylase 1 & ACACA & Bacterial Infections \\
\hline Q16539 & Mitogen-activated protein kinase 14 & MAPK14 & Endotoxemia; Inflammation; \\
\hline Q16881 & Thioredoxin reductase, cytoplasmic & TXNRD1 & $\begin{array}{l}\text { Bacterial Infections; Oxidative and free radical } \\
\text { damage; Pneumocystis infections; }\end{array}$ \\
\hline P45983 & Mitogen-activated protein kinase 8 & MAPK8 & Inflammatory Disorders, Unspecified \\
\hline P16581 & E-selectin & SELE & Chronic Obstructive Pulmonary Disease (COPD); \\
\hline P13726 & Tissue factor & F3 & Sepsis \\
\hline P04054 & Phospholipase A2 & PLA2G1B & Inflammation \\
\hline P07858 & Cathepsin B & CTSB & Acute otitis media \\
\hline P22894 & Neutrophil collagenase & MMP8 & Inflammatory diseases \\
\hline 014920 & $\begin{array}{l}\text { Inhibitor of nuclear factor kappa B } \\
\text { kinase beta subunit }\end{array}$ & IKBKB & Inflammation; Inflammatory lung disease \\
\hline P14416 & $\mathrm{D}(2)$ dopamine receptor & DRD2 & Nausea and vomiting; Respiratory diseases \\
\hline P50406 & 5-hydroxytryptamine 6 receptor & HTR6 & Nausea and vomiting \\
\hline P41143 & Delta-type opioid receptor & OPRD1 & Cough \\
\hline P45984 & Mitogen-activated protein kinase 9 & MAPK9 & Inflammation \\
\hline P24941 & Cell division protein kinase 2 & CDK2 & Viral infection, unspecified \\
\hline \multirow[t]{6}{*}{ P08311 } & Cathepsin G & CTSG & Chronic Obstructive Pulmonary Disease (COPD) \\
\hline & Beta-lactamase & & Bacterial Infections \\
\hline & DNA polymerase (HSV) & & Herpes virus infection; Viral infection, unspecified \\
\hline & P-hydroxybenzoate hydroxylase & & Inflammation \\
\hline & Rhinovirus coat protein & & Acute nasopharyngitis (common cold) \\
\hline & Streptavidin & & Bacterial Infections \\
\hline
\end{tabular}


DNA polymerase (HSV), encoded by herpes simplex virus (HSV), can selectively replicate viral DNA through a variety of mechanisms. Rhinovirus coat protein is thought to cause Rhinoviral infection. It indicates that YD may cure bacterial infections through decreasing drug resistance by acting as natural inhibitors of $\beta$ lactamases, and play a role of antivirus by inhibiting DNA polymerase and rhinovirus coat protein.

\section{Target-target interaction (T-T) networks}

The protein interaction network of 33 target genes was constructed using STRING (Figure 1). Genes were denoted as nodes and interactions between gene pairs were presented as edges (lines) in the image. A total of 33 nodes and 127 edges composed the acquired interaction network. The degree of each node is shown in Table 2. Average node degree and the clustering coefficient were 7.7 and 0.617 , respectively for this network having no PPI enrichment value. In the network, the degree of node (the number of connections or edges the node has with other nodes) is one of the most basic quantitative properties and the nodes with high degree are considered as hubs. Of the 33 proteins, 15 proteins possess node degree larger than 8 under an average value of 7.7 , therefore, these candidate proteins participating in more interactions than other proteins are the hubs in this T-T Network. In the network, TNF (Tumor necrosis factor) was the node with the highest degree $(\mathrm{DD}=19)$, followed by PTGS2 (Prostaglandin $\mathrm{G} / \mathrm{H}$ synthase 2 , DD $=18$ ), IL6 (Interleukin-6, DD = 17), IL1B (Interleukin-1 beta, DD = 14), MAPK14 (Mitogen-activated protein kinase 14, DD = 14), PPARG (Peroxisome proliferator activated receptor gamma, $\mathrm{DD}=13$ ), VEGFA (Vascular endothelial growth factor A, $\mathrm{DD}=13$ ), CCL2 (C-C motif chemokine 2, DD = 12), NOS3 (Nitric-oxide synthase, endothelial, $D D=12$ ) and MAPK8 (Mitogen-activated protein kinase $8, D D=12$ ). Most of these proteins show close relationships with inflammation. In addition, OPRD1 (Delta-type opioid receptor, DD = 4), DRD2 $(\mathrm{D}(2)$ dopamine receptor, $\mathrm{DD}=3)$, CHRM2 (Muscarinic acetylcholine receptor M2, DD = 5) and OPRM1 (Mu-type opioid receptor, $\mathrm{DD}=5$ ) showed a closely interaction with each other and may play a special role in this network also.

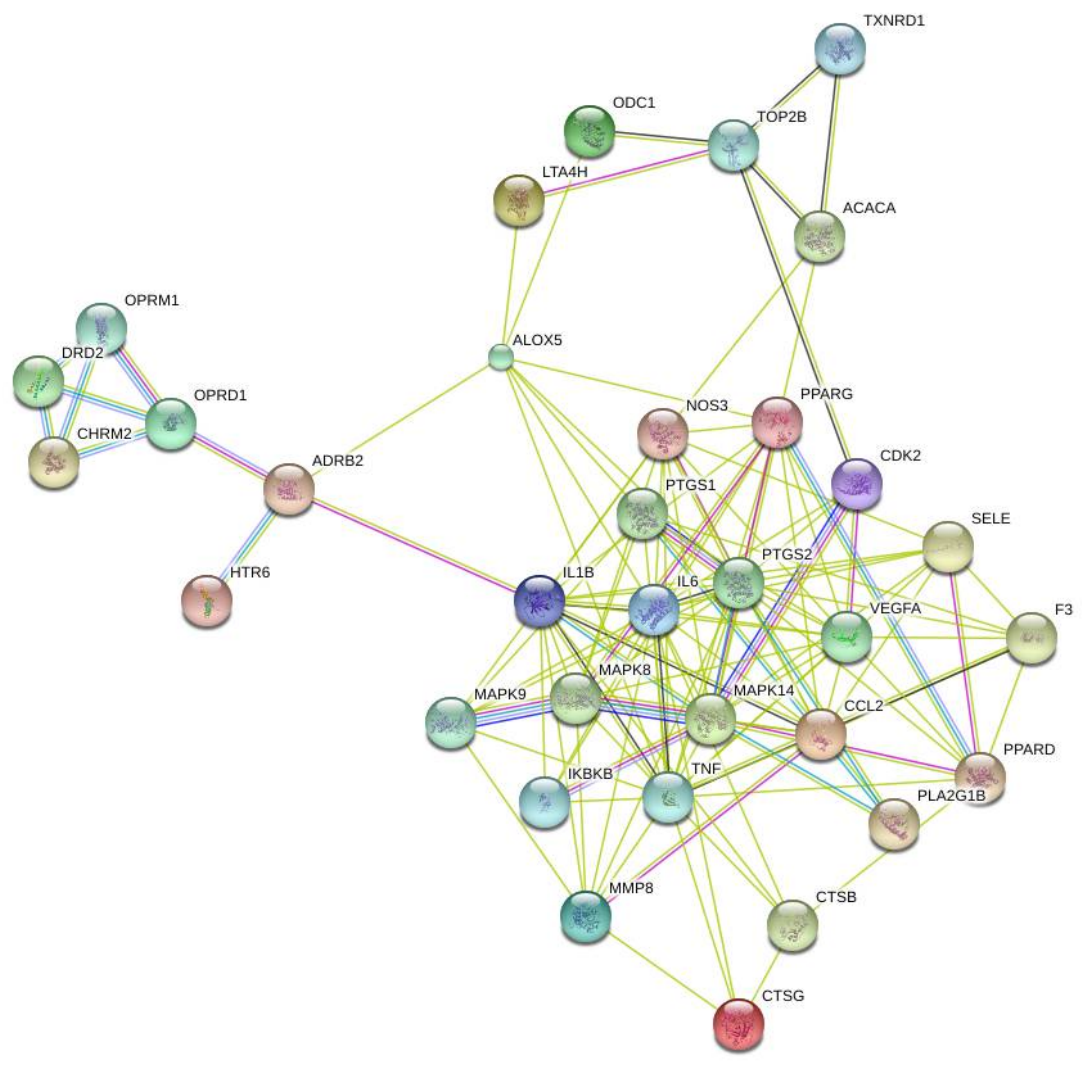

Figure 1: Protein interaction networks based on search tool for the retrieval of interacting genes/proteins database. Genes are denoted as nodes and interactions between gene pairs are presented as edges (lines) in the image. A total of 33 nodes and 127 edges constitute this interaction network 


\section{Herb-target networks}

For further studying the relationship between targets and herbs, we constructed herb-target network using Cytoscape (Figure2). The information of the related targets-herbs are shown in Table 3. In the network, PTGS2,
PTGS1, IL6, NOS3, ADRB2, TNF, TOP2 and CHRM2 were the nodes with the highest degree (DD = 7), followed by PPARG, LTA4H, ALOX5 and PPARD (DD = 6), CDK2, CCL2, IL1B, VEGFA, OPRM1, ODC1, ACACA (DD = 5), MAPK14, TXNRD1, MAPK8, SELE and F3 (DD $=4)$.

Table 2: The degree of each node present in network

\begin{tabular}{lccrcc}
\hline Node & Degree & Node & Degree & Node & Degree \\
\hline TNF & 19 & ALOX5 & 8 & ACACA & 4 \\
PTGS2 & 18 & PPARD & 8 & ADRB2 & 4 \\
IL6 & 17 & PTGS1 & 8 & OPRD1 & 4 \\
IL1B & 14 & MMP8 & 8 & CTSG & 4 \\
MAPK14 & 14 & F3 & 7 & OPRM1 & 3 \\
PPARG & 13 & MAPK9 & 7 & PLA2G1B & 3 \\
VEGFA & 13 & TOP2B & 5 & DRD2 & 3 \\
CCL2 & 12 & CHRM2 & 5 & LTA4H & 2 \\
NOS3 & 12 & CTSB & 5 & ODC1 & 2 \\
MAPK8 & 12 & IKBKB & 5 & TXNRD1 & 2 \\
SELE & 9 & CDK2 & 5 & HTR6 & 1 \\
\hline
\end{tabular}

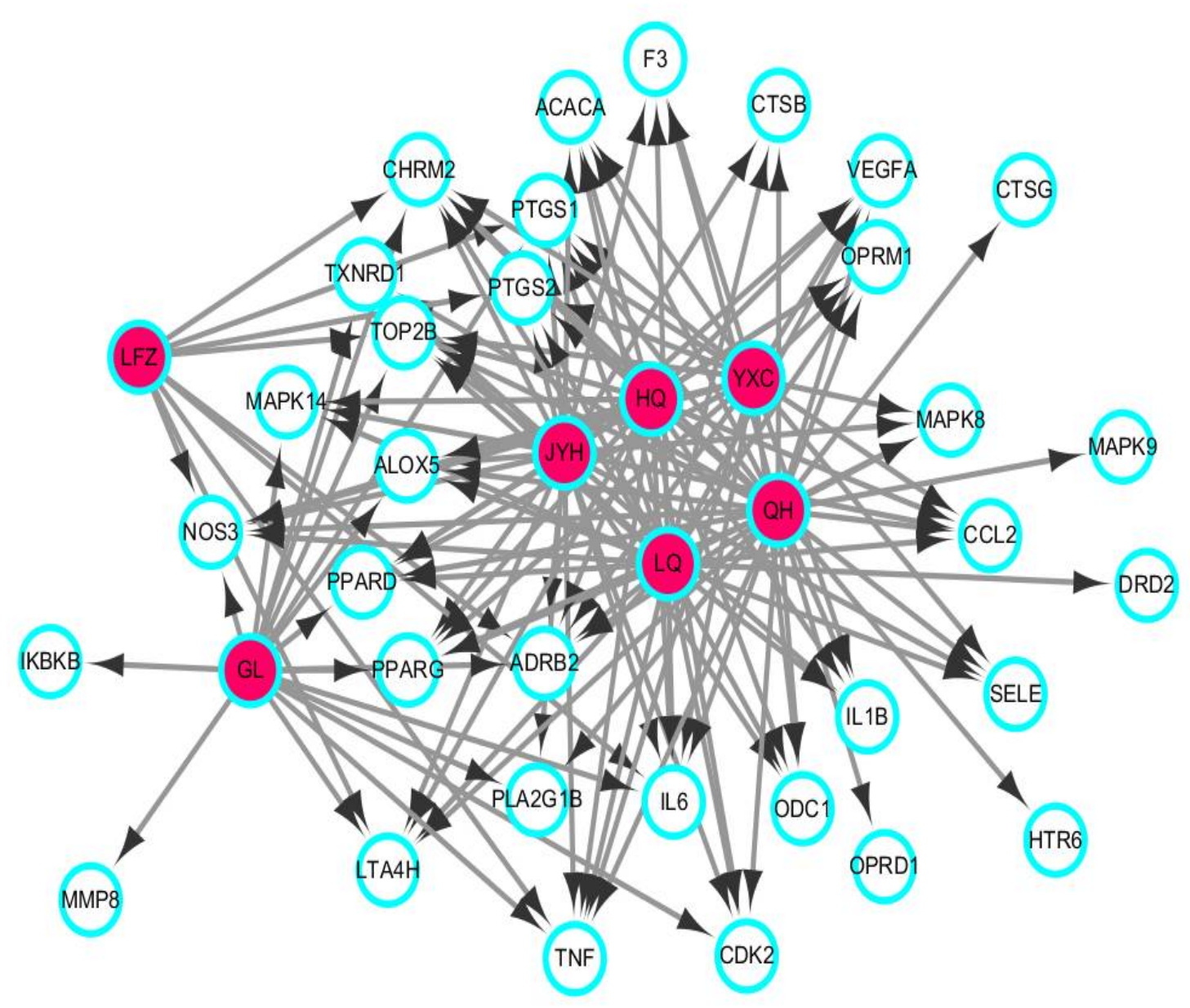

Figure 2: Herb-target networks. The red circles represent herbs in YD, while the white circles represent target proteins, and each edge represents the interaction between them. 
Table 3: Target-herb relationship

\begin{tabular}{|c|c|c|c|c|c|}
\hline Target & Degree & Herb & Target & Degree & Herb \\
\hline PTGS2 & 7 & GL;HQ;JYH;LFZ;LQ;QH;YXC & MAPK14 & 4 & GL;HQ;JYH;LQ \\
\hline PTGS1 & 7 & GL;HQ;JYH;LFZ;LQ;QH;YXC & TXNRD1 & 4 & GL;JYH;LQ;QH \\
\hline IL6 & 7 & GL;HQ;JYH;LFZ;LQ;QH;YXC & MAPK8 & 4 & JYH;LQ;QH;YXC \\
\hline NOS3 & 7 & GL;HQ;JYH;LFZ;LQ;QH;YXC & SELE & 4 & JYH;LQ;QH;YXC \\
\hline ADRB2 & 7 & GL;HQ;JYH;LFZ;LQ;QH;YXC & F3 & 4 & JYH;LQ;QH;YXC \\
\hline TNF & 7 & GL;HQ;JYH;LFZ;LQ;QH;YXC & PLA2G1B & 3 & GL;JYH;QH \\
\hline TOP2 & 7 & GL;HQ;JYH;LFZ;LQ;QH;YXC & CTSB & 3 & JYH;LQ;QH \\
\hline CHRM2 & 7 & GL;HQ;JYH;LFZ;LQ;QH;YXC & MMP8 & 1 & $\mathrm{GL}$ \\
\hline PPARG & 6 & GL;HQ;JYH;LQ;QH;YXC & IKBKB & 1 & $\mathrm{GL}$ \\
\hline LTA4H & 6 & GL;HQ;JYH;LFZ;LQ;QH & DRD2 & 1 & LQ \\
\hline ALOX5 & 6 & GL;HQ;JYH;LQ;QH;YXC & HTR6 & 1 & $\mathrm{QH}$ \\
\hline PPARD & 6 & GL;HQ;JYH;LQ;QH;YXC & OPRD1 & 1 & $\mathrm{QH}$ \\
\hline CDK2 & 5 & GL;HQ;JYH;LQ;QH & MAPK9 & 1 & $\mathrm{QH}$ \\
\hline CCL2 & 5 & $\mathrm{HQ} ; \mathrm{JYH} ; \mathrm{LQ} ; \mathrm{QH} ; \mathrm{YXC}$ & CTSG & 1 & $\mathrm{QH}$ \\
\hline IL1B & 5 & HQ;JYH;LQ;QH;YXC & Beta-lactamase & 6 & GL;HQ;JYH;LFZ;LQ;QH \\
\hline VEGFA & 5 & HQ;JYH;LQ;QH;YXC & $\begin{array}{l}\text { Rhinovirus coat } \\
\text { protein }\end{array}$ & 2 & GL;JYH \\
\hline OPRM1 & 5 & HQ;JYH;LQ;QH;YXC & $\begin{array}{l}\text { DNA polymerase } \\
\text { (HSV) }\end{array}$ & 2 & $\mathrm{GL} ; \mathrm{QH}$ \\
\hline ODC1 & 5 & HQ;JYH;LQ;QH;YXC & $\begin{array}{l}\text { P-hydroxybenzoate } \\
\text { hydroxylase }\end{array}$ & 1 & JYH \\
\hline ACACA & 5 & HQ;JYH;LQ;QH;YXC & Streptavidin & 1 & $\mathrm{QH}$ \\
\hline
\end{tabular}

Note: $\mathrm{GL}=$ Trichosanthes kirilowii Maxim; $\mathrm{HQ}=$ Scutellariae radix; $\mathrm{LQ}=$ Forsythiae fructus; $\mathrm{JYH}=$ Lonicerae japonicae Flos; LFZ = Raphani semen; $\mathrm{QH}=$ Peucedani radix; YXC = Houttuyniae herba

\section{Go and pathway analysis data}

To analyze biological functions of these potential targets, GO and Pathway enrichment analysis was conducted. We obtained 576 GO terms (Biological Process) and 75 KEGG pathways (False Discovery Rate, FDR < 0.05) (Table 4). It is interesting to note that these targets are involved in a variety of biological processes including response to oxygen-containing compound and regulation of cell proliferation, response to bacterium. These biological processes are largely related to transcriptional regulation, immune response, and apoptosis. It indicates that these biological processes have a close relationship with inflammation. Of 75 KEGG pathway terms, TNF signaling pathway, NOD-like receptor signaling pathway, influenza $A$ and toll-like receptor signaling pathway have the lowest FDR. Four pathways are associated with virus infection, immune tolerance, immune response and inflammatory response, which play an important role in respiratory infection diseases.

\section{DISCUSSION}

YD is effective in amiolerating lung condition, relieving exterior syndrome, relaxing the bowels and removing food retention, and has been used for treating pediatric diseases, especially pneumonia and recurrent respiratory tract infection [9-14]. However, the precise mechanisms of YD action in these diseases are still unclear. Thus, system pharmacology method combining the screening drug targeting, network construction, and pathway analysis was carried out in this work to uncover the active ingredients, targets, and pathways of YD and systematically deciphered its therapeutic mechanism of actions [15]. Our results showed that 627 ingredients and 654 potential targets were obtained from YD, and 37 targets were screened out. GO analysis of these targets and integrated herb-target network analysis demonstrated the synergistic e $\square$ ect of YD ingredients in treating the related diseases mainly through boosting of immune system, inhibiting inflammatory response, and inhibiting/killing pathogens as well as decreasing drug resistance [15]. Meanwhile, the pathway analysis in our work shows that YD might simultaneously regulate multitargets/pathways coupled with a range of therapeutic modules, for example, the suppression of inflammation and virus/bacterial infections and decreasing drug resistance [12]. Previous studies have shown that YD could boost immune system, inhibit inflammatory response and ease the damage caused by inflammation through reduction of expression of IL- 6 and TNF- $\alpha$ and increasing the expression of IL-2, IL-10, IFN- $\gamma$, SIgA and IgM [3]. In this study, GO enrichment analysis, network analysis and pathway analysis exhibited that YD significantly enriches target genes involved in reducing the inflammation response, enhancing immunity and combating viral/bacterial growth. 
Table 4: Part of the GO terms and pathway terms of the potential targets

\begin{tabular}{|c|c|c|c|c|}
\hline Type & ID & Term & Gene count & FDR \\
\hline \multirow{23}{*}{ 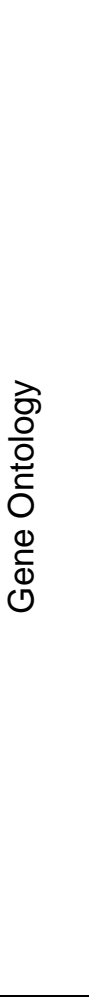 } & GO:1901700 & Response to oxygen-containing compound & 19 & $2.20 \mathrm{E}-11$ \\
\hline & GO:0009612 & Response to mechanical stimulus & 11 & 2.47E-11 \\
\hline & G0.0033993 & Response to lipid & 16 & 2.47E-11 \\
\hline & G0.0010033 & Response to organic substance & 22 & $1.25 \mathrm{E}-10$ \\
\hline & G0.0010243 & Response to organonitrogen compound & 15 & $2.54 \mathrm{E}-10$ \\
\hline & G0.0042127 & Regulation of cell proliferation & 18 & 3.84E-10 \\
\hline & GO.0044707 & Single-multicellular organism process & 28 & 3.84E-10 \\
\hline & GO.0051707 & Response to other organism & 14 & 4.10E-10 \\
\hline & GO.1901698 & Response to nitrogen compound & 15 & 4.99E-10 \\
\hline & GO.0071310 & Cellular response to organic substance & 19 & $6.43 \mathrm{E}-10$ \\
\hline & G0.0009628 & Response to abiotic stimulus & 16 & $6.92 \mathrm{E}-10$ \\
\hline & GO.0051240 & $\begin{array}{l}\text { Positive regulation of multicellular organismal } \\
\text { process }\end{array}$ & 17 & $6.92 \mathrm{E}-10$ \\
\hline & GO.0097305 & Response to alcohol & 11 & $7.84 \mathrm{E}-10$ \\
\hline & G0.0008284 & Positive regulation of cell proliferation & 14 & 2.12E-09 \\
\hline & GO.0051049 & Regulation of transport & 18 & 2.18E-09 \\
\hline & G0.0032496 & Response to lipopolysaccharide & 10 & 5.19E-09 \\
\hline & G0.0051050 & Positive regulation of transport & 14 & 5.65E-09 \\
\hline & GO.0009605 & Response to external stimulus & 18 & $6.79 \mathrm{E}-09$ \\
\hline & G0.0070482 & Response to oxygen levels & 10 & 8.56E-09 \\
\hline & GO.0009719 & Response to endogenous stimulus & 16 & 1.27E-08 \\
\hline & GO.0032879 & Regulation of localization & 19 & 1.27E-08 \\
\hline & GO.0023056 & Positive regulation of signaling & 16 & 1.79E-08 \\
\hline & GO.0009617 & Response to bacterium & 11 & 1.96E-08 \\
\hline \multirow{10}{*}{ 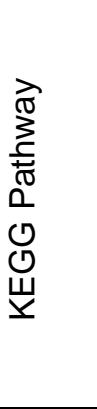 } & 4668 & TNF signaling pathway & 9 & $1.90 \mathrm{E}-11$ \\
\hline & 4621 & NOD-like receptor signaling pathway & 7 & 4.53E-10 \\
\hline & 5164 & Influenza A & 7 & 5.24E-07 \\
\hline & 4620 & Toll-like receptor signaling pathway & 6 & 5.93E-07 \\
\hline & 590 & Arachidonic acid metabolism & 5 & $1.59 \mathrm{E}-06$ \\
\hline & 5133 & Pertussis & 5 & 2.65E-06 \\
\hline & 4080 & Neuroactive ligand-receptor interaction & 7 & 5.69E-06 \\
\hline & 5168 & Herpes simplex infection & 6 & 7.02E-06 \\
\hline & 4370 & VEGF signaling pathway & 4 & 4.30E-05 \\
\hline & 4064 & NF-kappa B signaling pathway & 4 & 0.000155 \\
\hline
\end{tabular}

\section{CONCLUSION}

The results of this study describe important molecular targets and signaling pathways of YD. It is concluded that the mechanisms of YD for related diseases mainly include restoring the immune system and enhancing immune response, alleviating the symptoms of inflammation disorders, and combating the spreading virus/bacterial. This study not only made a contribution to a better understanding of the mechanisms of $\mathrm{YD}$, but also proposed a strategy to develop novel TCM candidates at a network pharmacology level. However, this study contains only those ingredients and targets of YD that have been published in literature. Thus, further studies such as docking and MD simulations are needed to verify the validity of the results.

\section{DECLARATIONS}

\section{Acknowledgement}

This work was financially supported by National Natural Science Foundation of China (no. 81403328) and Beijing Educational Committee Cooperation Project, China (no.1000062520115).

\section{Conflict of Interest}

No conflict of interest associated with this work.

\section{Contribution of Authors}

The authors declare that this work was done by the authors named in this article and all liabilities pertaining to claims relating to the content of this article will be borne by them. Tie-Gang Liu, Zi-An 
Zheng and Chen Bai contributed equally to this article.

\section{Open Access}

This is an Open Access article that uses a funding model which does not charge readers or their institutions for access and distributed under the terms of the Creative Commons Attribution License (http://creativecommons.org/licenses/by/ 4.0) and the Budapest Open Access Initiative (http://www.budapestopenaccessinitiative.org/rea d), which permit unrestricted use, distribution, and reproduction in any medium, provided the original work is properly credited.

\section{REFERENCES}

1. Lv G, Yu H, Gu X. Case Series Reports of 40 Pediatric Cold Children with Lung-stomach Heat Retention Syndrome Treated by Yinlai Decoction Addition and Subtraction. J Zhejiang Chinese Med Uni 2014; 38: 973975.

2. Gu $X, Y u H$. Influence of Yinlai Decoction on serum IL-2 and TNF- $\alpha$ levels in mice with lung-stomach heat retention syndrome. J Beijng Uni Trad Chinese Med 2008; 33: 5754-5776.

3. Liu T, Yu H, Zhang $W, L v G$, Zhen J, Li X, Gu X. Influences of Yinlai Decoction on slgA, TNF- $\alpha$ and IL-10 in intestinal mucosal tissues of mice with dyspepsia combined with influenza virus infection. J Beijng Uni Trad Chinese Med 2014; 37: 86-89.

4. Zhang W, Liu T, Zhen J, Lv G, Li X, Yu H, Gu X. Influence of Yinlai Decoction on immunity in mice with dyspepsia combining influenza virus infection. J Beijing Uni Trad Chinese Med 2014; 37: 543-547.

5. Wu XM, Wu CF. Network pharmacology: a new approach to unveiling Traditional Chinese Medicine. Chin J Nat Med 2015; 13: 1-2.

6. Ru J, Li P, Wang J, Zhou W, Li B, Huang C, Li P, Guo Z, Tao W, Yang Y, Xu X, Li Y, Wang Y, Yang L. TCMSP: a database of systems pharmacology for drug discovery from herbal medicines. J Cheminform 2014; 6: 13-19.

7. Szklarczyk D, Franceschini A, Wyder S, Forslund K, Heller D, Huerta-Cepas J, Simonovic M, Roth A, Santos A, Tsafou KP, Kuhn M, Bork $P$, Jensen LJ, von Mering C. STRING v10: protein-protein interaction networks, integrated over the tree of life. Nucleic Acids Res. 2015; 43: D447-D452.

8. Shannon P, Markiel A, Ozier O, Baliga NS, Wang JT, Ramage $D$, Amin $N$, Schwikowski B, ldeker $T$. Cytoscape: a software environment for integrated models of biomolecular interaction networks. Genome Res 2003; 13: 2498-2504.

9. Consortium, Uni Prot. UniProt: a hub for protein information. Nucleic Acids Res 2015; 43: 204-212.

10. Pratt RF. Beta-Lactamases: Why and How. J Med Chem 2016; 59: 8207-8220.

11. Diniz YJ, Girão $A M$, Zaccur $L K$, Costa $S F$, Netto $B P$, Leonardo B, Peter SR, Bicca DR, Cláudia CA, Cecília $D M B V$, Vitor FF, Viveca $A G$, Cláudio $C$, lzabel $C P$. Docking of anti-HIV-1 oxoquinoline - acylhydrazone derivatives as potential HSV-1 DNA polymerase inhibitors. J Mol Structure 2014; 1074: 263-270.

12. Rollinger JM, Steind TM, Schuster DO, Kirchmair J, Anrain K, Ellmerer EP, Langer T, Stuppner H, Wutzler $P$, Schmidtke M. Structure-based virtual screening for the discovery of natural inhibitors for human rhinovirus coat protein. J Med Chem. 2008; 51: 842-851.

13. Zhang J, Miao J, Hou J, Lu C. The effects of H3N2 swine influenza virus infection on TLRs and RLRs signaling pathways in porcine alveolar macrophages. Virol $J$ 2015; 12: 76-81.

14. Dou Y, Zhao Y, Zhang ZY, Mao HW, Tu WW, Zhao XD. Respiratory syncytial virus infection induces higher tolllike receptor-3 expression and TNF- $\alpha$ production than human metapneumovirus infection. Plos One 2013; 8: e73488-e73488.

15. Xiao YT, Li GX, Wang XM. Effect mechanism of NOD like receptor signaling pathway on intestinal mucosal barrier of rat during early phase of acute intra-abdominal infection]. Zhonghua Wei Zhong Bing Ji Jiu Yi Xue 2013; 25: 527-532. 